\title{
Confidence Intervals for Lower Quantiles Based on Two-Sample Scheme
}

\author{
H. Morabbi, M. Razmkhah* and J. Ahmadi \\ Ferdowsi University of Mashhad
}

\begin{abstract}
In this paper, a new two-sampling scheme is proposed to construct appropriate confidence intervals for the lower population quantiles. The confidence intervals are determined in the parametric and nonparametric set up and the optimality problem is discussed in each case. Finally, the proposed procedure is illustrated via a real data set.
\end{abstract}

Keywords. Order statistics; coverage probability; optimality; expected width; exponential distribution.

MSC 2010: 62G15 (62G30, 62D05).

\section{Introduction and Preliminaries}

Let $X_{1}, X_{2}, \ldots, X_{n}$ be independent and identically distributed (iid) continuous random variables with cumulative distribution function (cdf) $F(\cdot)$ and probability density function (pdf) $f(\cdot)$ and denote the corresponding order statistics by $X_{1: n}, X_{2: n}, \ldots, X_{n: n}$. The order statistics arise in a wide variety of practical situations such as industrial stress testing and also play an important role in survival analysis and reliability theory. The interested readers may refer to David and Nagaraja (2003) and Arnold et al. (2008) for more details. Furthermore, the order statistics have been used in the nonparametric inferences about the population parameters, especially, when the quantiles are of interest. It is well-known that for any real number $p$ in the interval $(0,1)$, the $p$ th quantile of the $\operatorname{cdf} F(\cdot)$ is defined by $\xi_{p}=\inf \{x: F(x) \geqslant p\}$.

* Corresponding author 
The quantiles are used to construct the concentration and deviation criteria. In the context of quality control, they are also used to make decision about the warranty of a product. Several authors have studied the problem of constructing confidence intervals (CIs) for the quantiles based on various types of ordered data. Krewski (1976) obtained distribution-free CIs for quantile intervals on the basis of order statistics. Emerson (1982) found distributionfree CIs for the median in the presence of right censoring. Strawderman et al. (1997) and Guilbaud (2001) investigated the interval estimation of quantiles under random censoring and progressive Type-II censoring, respectively; see also, Chapter 7 of David and Nagaraja (2003) and Section 7.7 of Arnold et al. (2008). Razmkhah et al. (2008) obtained distribution-free CIs for the population quantiles on the basis of the minima and maxima in some independent samples. Distribution-free CIs for quantiles on the basis of record values and current $k$-records were derived by Ahmadi and Arghami (2003) and Ahmadi et al. (2009), respectively. Similar work has been done by Balakrishnan and Li (2006) based on ordered ranked set samples.

In the situations in which the measurement process is costly or destructive such as biomedical research, industrial experiments and life testing, it seems logical to do inference about the parameter of interest based on few observed data. So, an alternative scheme to simple random sampling (SRS) may be formed based on a sequential sampling method to record some special points. Such a scheme may lead to a measurement saving process. In this paper, we propose a two-sample scheme such that the target is attained on the basis of a data set of smaller number of observed data than that of a corresponding SRS scheme. In the first step an initial random sample of size $n$ is taken from the underlying distribution and then using the information obtained from this sample, a new sample of size $m$ is added to the available data set. That is, a set of observed data with size $n+m$ would be used in inference. It is of important to note that in the lifetime experiments, the units were not damaged by the testing, can be used in the future experiments or other purposes. Let us now assume that $X_{1}, X_{2}, \ldots, X_{r}$ are iid random variables from the cdf $F$. In order to construct a two-sided CI for $\xi_{p}=F^{-1}(p)$ based on SRS, the coverage probability $(\mathrm{CP})$ of the event $\left\{X_{i_{1}: r} \leqslant \xi_{p} \leqslant X_{i_{2}: r}\right\}$ is given by

$$
\gamma_{1}\left(i_{1}, i_{2}, r, p\right)=\sum_{k=i_{1}}^{i_{2}-1}\left(\begin{array}{l}
r \\
k
\end{array}\right) p^{k}(1-p)^{r-k} .
$$

In addition, it is well-known that $\left(X_{1: r}, X_{r: r}\right)$ is a two-sided CI for $\xi_{p}$ with 
the maximum coverage probability $1-p^{r}-(1-p)^{r}$, which is free of $F$; see Arnold et al. (2008, p. 183). For given $p$ and $\alpha$, the minimum sample size, denoted by $r_{0}$, needed to attain the confidence level $1-\alpha$ is given by

$$
r_{0}=\min \left\{r: 1-p^{r}-(1-p)^{r} \geqslant 1-\alpha\right\} .
$$

Moreover, the values of $i_{1}$ and $i_{2}$, for which $i_{2}-i_{1}$ is minimum, may also be determined and denoted by $i_{1}^{*}$ and $i_{2}^{*}$ such that $\left(X_{i_{1}^{*}: r_{0}}, X_{i_{2}^{*}: r_{0}}\right)$ is a $95 \%$ CI for $\xi_{p}$. The corresponding exact CPs are also computed by the use of (1). For $\alpha=0.05$, the values of $r_{0}, i_{1}^{*}, i_{2}^{*}$ and CPs are numerically calculated and the results are presented in Table 1 for some selected values of $p \leqslant 0.5$. Note that by symmetry, the results for $p>0.5$ are similarly deduced.

Table 1. Values of minimum sample size to construct a $95 \%$ CI for $\xi_{p}$.

\begin{tabular}{cccccccccccc}
\hline \hline$p$ & 0.025 & 0.05 & 0.1 & 0.15 & 0.2 & 0.25 & 0.3 & 0.35 & 0.4 & 0.45 & 0.5 \\
\hline$r_{0}$ & 119 & 59 & 29 & 19 & 14 & 11 & 9 & 7 & 7 & 6 & 6 \\
$i_{1}^{*}$ & 1 & 1 & 1 & 1 & 1 & 1 & 1 & 1 & 1 & 1 & 1 \\
$i_{2}^{*}$ & 10 & 10 & 9 & 8 & 8 & 7 & 7 & 7 & 6 & 6 & 6 \\
$C P$ & 0.9500 & 0.9509 & 0.9526 & 0.9503 & 0.9536 & 0.9502 & 0.9554 & 0.9503 & 0.9532 & 0.9640 & 0.9688 \\
\hline
\end{tabular}

From Table 1, it is observed that for lower quantiles a moderately large simple random sample is needed to construct a 95\% CI, while for central quantiles the required sample size is rather small. Therefore, to construct CIs for the lower quantiles, the proposed sampling scheme may be used instead of SRS. In this case, it seems reasonable to record the observations that are less than one of intermediate order statistics of the first sample. In general, depending on the main goal of the inference, some restrictions can be considered to obtain the second sample.

The rest of this paper is organized as follows. In Section 2, the model of interest is described and some auxiliary results are presented. Also distributionfree CIs for lower quantiles are constructed. The optimality problem is investigated in Section 3. To illustrate the proposed procedure in the paper a real data set is used in Section 4. Finally, some conclusions are stated.

\section{Model Description and CIs}

In a number of experiments, such as in industrial stress testing and other similar situations, measurements may be made sequentially and only values smaller than a previous specific point are recorded. This sampling can be 
important when the measurement process is costly or destructive. Now, let $X_{1}, \ldots, X_{n}$ be an initial random sample from the $\operatorname{cdf} F(\cdot)$ and $\left\{X_{i}^{\prime}, i \geqslant 1\right\}$ be another sequence of iid random variables from the same distribution. To obtain the second sample, we record the first $m$ observations from the sequence $\left\{X_{i}^{\prime}, i \geqslant 1\right\}$ which for fixed $j$, they are less than the observed value of the $j$ th order statistic of the initial sample $x_{j: n}$. That is, we take a random sample of size $m$ from the same distribution as $X_{i}^{\prime} \mid X_{i}^{\prime} \leqslant x_{j: n}$. For estimating $\xi_{p}$, a proper choice for $j$ may be approximately determined as $[n p]$, where $[x]$ denotes the integer part of $x$. This sampling scheme is useful in many practical situation. For example, consider an experiment in which the breaking stress of some wooden beams should be measured for inference about the strength of such beams. So far, the methods of simple random sampling and record sampling have been studied in this situation; see, for example, Glick (1978) or Gulati and Padgett (1994, 2003). Here, we produce a two-sampling scheme to obtain a suitable data set. At the first step, $n$ beams are selected at random and their strengths are measured to attain an initial sample. At the second step, some beams are sequentially tested to obtain $m$ new data points as the second sample. In this step, a beam is selected at random and stressed up to the observed value of breaking stress of the $j$ th smallest strength of the initial sample. If it breaks, the breaking stress point of that beam is recorded as a new data point. Otherwise, it comes back to storehouse for after usage and another beam is selected. Also, it must be noted that under this process, the beams which are not destroyed, have additional information that the strengths of them are greater than the $j$ th order statistic of the initial sample which can be useful in the future experiment. This process is sequentially done until $m$ new data points are observed.

Suppose that for given $j(1 \leqslant j \leqslant n), Y_{1}^{(j)}, \ldots, Y_{m}^{(j)}$ is a random sample of size $m$ from the right-truncated distribution at the observed value of the $j$ th order statistic of the initial sample, $x_{j: n}$. So, the data set in the two-sampling scheme is given by $\left\{Z_{1}^{(j)}, \ldots, Z_{n+m}^{(j)}\right\}$ for which

$$
Z_{1}^{(j)}=X_{1}, \ldots, Z_{n}^{(j)}=X_{n} \quad \text { and } \quad Z_{n+1}^{(j)}=Y_{1}^{(j)}, \ldots, Z_{n+m}^{(j)}=Y_{m}^{(j)},
$$

where $X_{1}, \ldots, X_{n}$ represents the initial sample. Let $Z_{r: n+m}^{(j)}$ denote the $r$ th order statistic of the $Z^{(j)}$-sample, then we readily find that

$$
Z_{r: n+m}^{(j)}= \begin{cases}X_{r-m: n}, & r \geqslant m+j, \\ T_{r: m+j-1}, & r \leqslant m+j-1,\end{cases}
$$


where $T_{r: m+j-1}$ is the $r$ th order statistic among the random variables $\left\{X_{1: n}\right.$, $\left.\ldots, X_{j-1: n}, Y_{1: m}^{(j)}, \ldots, Y_{m: m}^{(j)}\right\}$. Therefore, the cdf of $Z_{r: n+m}^{(j)}$, for $r \geqslant m+j$, is given by

$$
P\left(Z_{r: n+m}^{(j)} \leqslant z\right)=\sum_{k=r-m}^{n}\left(\begin{array}{l}
n \\
k
\end{array}\right)[F(z)]^{k}[\bar{F}(z)]^{n-k} .
$$

For other cases, the result is presented in the following lemma.

Lemma 1. (Morabbi et al., 2015) For $r \leqslant m+j-1$, the cdf of $Z_{r: n+m}^{(j)}$ is given by

$$
\begin{aligned}
P\left(Z_{r: n+m}^{(j)} \leqslant z\right)= & F_{j: n}(z)+j\left(\begin{array}{c}
n \\
j
\end{array}\right) \sum_{i=r}^{m+j-1} \sum_{k=0}^{m+j-1-i n-j} \sum_{t=0}^{m}(-1)^{k+t}\left(\begin{array}{c}
m+j-1-i \\
k
\end{array}\right) \\
& \times\left(\begin{array}{c}
n-j \\
t
\end{array}\right)\left(\begin{array}{c}
m+j-1 \\
i
\end{array}\right)[F(z)]^{i+k} J_{j, i, k, t}(F(z)),
\end{aligned}
$$

where $F_{j: n}(z)$ stands for the cdf of $X_{j: n}$ and

$$
J_{j, i, k, t}(z)= \begin{cases}\frac{1-z^{t+j-(i+k)}}{t+j-(i+k)}, & t \neq i+k-j \\ -\log z, & t=i+k-j\end{cases}
$$

Since the cdf of $Z_{r: n+m}^{(j)}$ is a function of $F(\cdot)$, it is reasonable to construct CIs for the lower quantiles of $F$ on the basis of the data set $\left\{Z_{1}^{(j)}, \ldots, Z_{n+m}^{(j)}\right\}$.

Using (4), for $r \geqslant m+j$, it can be deduced that

$$
\eta_{2}(r, j, n, m, p)=P\left(Z_{r: n+m}^{(j)} \leqslant \xi_{p}\right)=\sum_{k=r-m}^{n}\left(\begin{array}{l}
n \\
k
\end{array}\right) p^{k}(1-p)^{n-k}
$$

Moreover for $r \leqslant m+j-1$, from (5), we get

$$
\begin{aligned}
\eta_{2}(r, j, n, m, p)= & P\left(Z_{r: n+m}^{(j)} \leqslant \xi_{p}\right) \\
= & \sum_{k=j}^{n}\left(\begin{array}{c}
n \\
k
\end{array}\right) p^{k}(1-p)^{n-k}+j\left(\begin{array}{c}
n \\
j
\end{array}\right) \sum_{i=r}^{m+j-1} \sum_{k=0}^{m+j-1-i} \sum_{t=0}^{n-j}(-1)^{k+t} \\
& \times\left(\begin{array}{c}
m+j-1-i \\
k
\end{array}\right)\left(\begin{array}{c}
n-j \\
t
\end{array}\right)\left(\begin{array}{c}
m+j-1 \\
i
\end{array}\right) p^{i+k} J_{j, i, k, t}(p),
\end{aligned}
$$


where $J_{j, i, k, t}(z)$ is as defined in (6). Therefore, the CP of the event $\left\{Z_{r_{1}: n+m}^{(j)} \leqslant\right.$ $\left.\xi_{p} \leqslant Z_{r_{2}: n+m}^{(j)}\right\}$ is given by

$$
\gamma_{2}\left(r_{1}, r_{2}, j, n, m, p\right)=\eta_{2}\left(r_{1}, j, n, m, p\right)-\eta_{2}\left(r_{2}, j, n, m, p\right),
$$

where $\eta_{2}(r, j, n, m, p)$ is as defined in (7) and (8) for $r \geqslant m+j$ and $r \leqslant$ $m+j-1$, respectively.

\section{Optimality}

As mentioned in Section 2, in obtaining the data set $\left\{Z_{1}^{(j)}, \ldots, Z_{n+m}^{(j)}\right\}$, two independent random samples are combined, for which to find the second sample, $m$ observations that are less than the observed value of $X_{j: n}$ are recorded from the sequence $\left\{X_{i}^{\prime}, i \geqslant 1\right\}$ all having the same $\operatorname{cdf} F(\cdot)$. Now, let us denote the number of needed units to observe the second sample by $K$, that is,

$$
K=\inf \left\{i: \sum_{t=1}^{i} I\left(X_{t}^{\prime} \leqslant X_{j: n}\right) \geqslant m\right\},
$$

where $I(A)$ stands for the indicator on the event $A$. It is not difficult to show that $K$ has negative binomial distribution with parameters $m$ and $p_{j}$, where

$$
p_{j}=P\left(X_{1}^{\prime} \leqslant X_{j: n}\right)=\frac{j}{n+1} .
$$

So, for given $p$, we first seek the optimal values for sample sizes of the first and second samples. Since the main goal of the proposed sampling scheme is to get the smallest number of observed data, one may find the optimal values of $(n, m)$, denoted by $\left(n^{*}, m^{*}\right)$, such that $n^{*}+m^{*}$ is minimum. These values are in fact the smallest integers satisfied $n \geqslant 2$ and $m \geqslant 1$, respectively. In addition to determining $\left(n^{*}, m^{*}\right)$, the problem of obtaining the optimal value for $j$, denoted by $j^{*}$, is of great interest. Therefore, we would like to obtain the 3-tuple $\left(n^{*}, m^{*}, j^{*}\right)$, which minimizes the expected value of total cost of the experiment with the following constraints

$$
\left\{\begin{array}{l}
\gamma_{2}(1, n+m, j, n, m, p) \geqslant 0.95, \\
j \geqslant \frac{m(n+1)}{a\left(r_{0}-n\right)},
\end{array}\right.
$$

where $\gamma_{2}(1, n+m, j, n, m, p)$ is as defined in (9), $r_{0}$ is the minimum sample size satisfying (2) with $\alpha=0.05$ and $a$ is a positive constant. It should be 
noted that the second condition in (10) is equivalent to $E(K) \leqslant a\left(r_{0}-n\right)$, where $E(K)$ stands for the expected value of $K$. Note that the total time on test $(T T T)$ associated with the proposed sampling scheme is given by

$$
T T T=(K-m) X_{j: n}+\sum_{i=1}^{n+m} Z_{i}^{(j)} .
$$

Let us now denote the costs of time and unit by $c_{t}$ and $c_{u}$, respectively. Then, the expected value of the total $\operatorname{cost}(E T C)$ is

$$
E T C=c_{t} E(T T T)+c_{u} E(K) .
$$

It is obvious that the $E T C$ is a decreasing function of $j$. Therefore, maximum $j$ satisfying (10) is the optimal $j$, say $j^{*}$. One can follow the algorithm given below to find values of $\left(n^{*}, m^{*}, j^{*}\right)$.

Algorithm 1. For given $p$, the optimal value of $(n, m, j)$ is determined through the following steps:

1. Set $n=2$ and $m=1$.

2. Take $s=n+m$.

3. For $j=1, \ldots, n$ check the conditions in (10).

4. If for one 3-tuple $(n, m, j)$, conditions (10) hold, then put $\left(n^{*}, m^{*}, j^{*}\right)=$ $(n, m, j)$. Else if more than one set of $(n, m, j)$ are found, then the one that imposes minimum expected value of $K$, is a reasonable choice for $\left(n^{*}, m^{*}, j^{*}\right)$.

5. If there is no any $j$ satisfied in conditions (10), put $s=s+1$.

6. Consider all pairs of $(n, m)$ such that $n+m=s ; n \geqslant 2, m \geqslant 1$, then go to step 3.

The final problem is to investigate which order statistics in the optimal data set $\left\{Z_{1}^{\left(j^{*}\right)}, \ldots, Z_{n^{*}+m^{*}}^{\left(j^{*}\right)}\right\}$ are the best to construct CI for $\xi_{p}$. In this direction, the values of $r_{1}^{*}$ and $r_{2}^{*}$ are numerically determined such that 
$\left(Z_{r_{1}^{*}: n^{*}+m^{*}}^{\left(j^{*}\right)}, Z_{r_{2}^{*}: n^{*}+m^{*}}^{\left(j^{*}\right)}\right)$ becomes the shortest width $95 \%$ CI for $\xi_{p}$. Exact CPs are also computed by the use of (9).

However, to compare two proposed procedures, one of the most important point is expected length. Therefore, it must be shown that for a fixed confidence level the expected length of the CI $\left(Z_{r_{1}^{*}: n^{*}+m^{*}}^{\left(j^{*}\right)}, Z_{r_{2}^{*}: n^{*}+m^{*}}^{\left(j^{*}\right)}\right.$, viz. $E\left(Z_{r_{2}^{*}: n^{*}+m^{*}}^{\left(j^{*}\right)}-Z_{r_{1}^{*}: n^{*}+m^{*}}^{\left(j^{*}\right)}\right.$, is shorter than the expected length of the CI based on SRS scheme, but it is obvious that the expected length of the CI depends on the underlying distribution. So, in the next subsection, we assume that $X$ has an exponential distribution and determine the optimal values of $n^{*}, m^{*}, j^{*}, r_{1}^{*}$ and $r_{2}^{*}$.

\subsection{Exponential Distribution}

Suppose that the underlying distribution is exponential with the $\operatorname{cdf} F(x)=$ $1-e^{-\theta x}, x>0, \theta>0$, where $\theta$ is the scale parameter. Without loss of generality, we take $\theta=1$. Now, let $\Delta_{1}\left(i_{1}, i_{2}, r_{0}\right)$ and $\Delta_{2}\left(r_{1}, r_{2}, n, m\right)$ be the expected length of the $100(1-\alpha) \%$ CIs based on SRS and two-sampling schemes, respectively. Therefore,

$$
\Delta_{1}\left(i_{1}, i_{2}, r_{0}\right)=\sum_{k=i_{1}}^{i_{2}-1} \frac{1}{r_{0}-k} .
$$

Moreover, by the use of (4), the expected value of $Z_{r: n+m}^{(j)}$, for $r \geqslant m+j$, is

$$
E\left(Z_{r: n+m}^{(j)}\right)=\sum_{i=0}^{r-m-1} \frac{1}{n-i} .
$$

Similarly, from (5), for $r \leqslant m+j-1$, we get

$$
\begin{aligned}
E\left(Z_{r: n+m}^{(j)}\right) & =\sum_{i=0}^{j-1} \frac{1}{n-i}-j\left(\begin{array}{c}
n \\
j
\end{array}\right) \sum_{i=r}^{m+j-1} \sum_{s=0}^{\infty}\left(\begin{array}{c}
m+j-1 \\
i
\end{array}\right) \\
& \times \operatorname{Beta}(j+s+1, n-j+1) \operatorname{Beta}(i+s+1, m+j-i),
\end{aligned}
$$

where $\operatorname{Beta}(\alpha, \beta)$ is the complete beta function. According to (12) and (13), the expected length of the CIs, $\left(Z_{r_{1}: n+m}^{(j)}, Z_{r_{2}: n+m}^{(j)}\right)$, is given by

$$
\Delta_{2}\left(r_{1}, r_{2}, n, m\right)=E\left(Z_{r_{2}: n+m}^{(j)}\right)-E\left(Z_{r_{1}: n+m}^{(j)}\right) .
$$


Now, for given $p$, we seek $j^{*}$, which minimizes the $E T C$ with the following constraints

$$
\left\{\begin{array}{l}
\gamma_{2}(1, n+m, j, n, m, p) \geqslant 0.95, \\
E(K) \leqslant a\left(r_{0}-n\right), \quad a>0, \\
\Delta_{2}\left(r_{1}, r_{2}, n, m\right) \leqslant \Delta_{1}\left(i_{1}, i_{2}, r_{0}\right)
\end{array}\right.
$$

where $\Delta_{1}\left(i_{1}, i_{2}, r_{0}\right)$ and $\Delta_{2}\left(r_{1}, r_{2}, n, m\right)$ are as defined in (11) and (14), respectively.

Under two sets of constraints (10) and (15), we have numerically determined the values of $n^{*}, m^{*}, j^{*}, r_{1}^{*}, r_{2}^{*}$ and also the CPs of the optimal CIs based on the proposed two-sampling scheme in the nonparametric and parametric (exponential distribution) set up, respectively. These are presented in Table 2 for some selected choices of $p$.

Table 2. Values of $n^{*}, m^{*}, j^{*}, r_{1}^{*}, r_{2}^{*}$ and the CPs for some choices of $p$ in a two-sampling scheme.

\begin{tabular}{|c|c|c|c|c|c|c|c|c|c|c|c|c|}
\hline \multirow[b]{2}{*}{$p$} & \multicolumn{5}{|c|}{ Nonparametric } & \multirow[b]{2}{*}{$C P$} & \multicolumn{6}{|c|}{ Exponential } \\
\hline & $n^{*}$ & $m^{*}$ & $j^{*}$ & $r_{1}^{*}$ & $r_{2}^{*}$ & & $n^{*}$ & $m^{*}$ & $j^{*}$ & $r_{1}^{*}$ & $r_{2}^{*}$ & $C P$ \\
\hline 0.025 & 15 & 9 & 1 & 1 & 13 & 0.9519 & 17 & 15 & 2 & 1 & 18 & 0.9513 \\
\hline 0.05 & 10 & 6 & 1 & 1 & 12 & 0.9500 & 10 & 12 & 2 & 1 & 15 & 0.9504 \\
\hline 0.10 & 7 & 4 & 1 & 1 & 8 & 0.9534 & 10 & 5 & 2 & 1 & 9 & 0.9522 \\
\hline 0.15 & 4 & 4 & 1 & 1 & 8 & 0.9510 & 7 & 6 & 3 & 1 & 10 & 0.9545 \\
\hline 0.20 & 5 & 2 & 1 & 1 & 7 & 0.9502 & 6 & 5 & 3 & 1 & 9 & 0.9555 \\
\hline
\end{tabular}

From Table 2, it is seen that for fixed value of $p$, the number of needed observed data based on the proposed two-sampling scheme, $n^{*}+m^{*}$, is less than that of SRS scheme $r_{0}$ (reported in Table 1), while the CPs of the both sampling schemes are greater than $95 \%$. Another point is that for exponential distribution, $j^{*}$ increases as $p$ increases.

\section{Illustrative Example}

In order to study the performance of the obtained results in the paper, we consider the failure time (in hours) of 38 conductors from Nelson and Doganaksoy (1995). The data are as follows:

$6.369,9.663,8.532,6.725,5.807,6.087,5.589,6.538,6.573,7.489,5.009$, $9.254,6.948,4.700,6.352,6.869,7.945,9.218,8.336,7.345,7.224,7.683$, 
$8.799,7.974,4.531,7.496,10.092,6.033,7.398,5.923,10.491,6.071,6.476$, $6.515,7.973,5.434,5.640,6.923$.

To find distribution-free CI based on SRS scheme, for example for $\xi_{0.1}$, according to the entire values in Table 1 , the first twenty-nine iid observations are considered and the $95 \% \mathrm{CI}$ is given by $\left(X_{1: 29}, X_{9: 29}\right)=(4.531,6.369)$, with exact $\mathrm{CP}$ equal to 0.9526 . Moreover, to construct distribution-free CI for $\xi_{0.1}$ on the basis of the proposed two-sampling scheme, by the use of Table 2, the first seven iid observations, i.e., 6.369, 9.663, 8.532, 6.725, 5.807, 6.087, 5.589 , are considered as an initial sample. Then the first four data points that are less than $X_{1: 7}=5.589$ are added to the available data set. Denoting the new observations by $Y_{1}^{(1)}, \ldots, Y_{4}^{(1)}$, the observed values are 5.009, 4.700, 4.531, 5.434. Therefore, the ordered data $Z_{1: 11}^{(1)}, \ldots, Z_{11: 11}^{(1)}$ are given by

$$
4.531,4.700,5.009,5.434,5.589,5.807,6.087,6.369,6.725,8.532,9.663 .
$$

Based on the later data set, the $95 \%$ distribution-free CI for $\xi_{0.1}$, with exact $\mathrm{CP}$ equal to 0.9500 , is $\left(Z_{1: 11}^{(1)}, Z_{8: 11}^{(1)}\right)=(4.531,6.369)$. It can be seen that the observed length of CIs based on SRS and proposed sampling scheme are the same, whereas, the observed units based on the new sampling scheme $\left(n^{*}+m^{*}=11\right)$ is too smaller than that of SRS $\left(r_{0}=29\right)$.

\section{Conclusions}

In this paper, we proposed a two-sampling scheme to obtain suitable data for constructing CIs for lower quntiles. Toward this end, we used two independent samples which were related together in distribution. The first sample was taken from the underlying population and the second sample was extracted from a right-truncated distribution at the observed value of the $j$ th order statistic of the initial sample. It was shown that the proposed sampling scheme would provide the CIs for lower quantiles with favorite CPs on the basis of a data set with smaller number of observed data and smaller expected length than that of SRS method. Hence, the proposed two-sampling scheme may be useful in many situations such as industry and lifetime experiments. We emphasize that in the lifetime experiments, while collecting the second sample the units which are not damaged by the testing, can be used in the future experiments or other purposes. 


\section{Acknowledgement}

We express our sincere thanks to the referees for their constructive suggestions and useful comments on the original version which resulted in this improved version of the paper.

\section{References}

Ahmadi, J. and Arghami, N.R. (2003). Nonparametric Confidence and Tolerance Intervals from Record Values Data, Statistical Papers, 44, 455-468.

Ahmadi, J., Razmkhah, M. and Balakrishnan, N. (2009). Current $k$-records and Their Use in Distribution-free Confidence Intervals, Statistics and Probability Letters, 79, 29-37.

Arnold, B.C., Balakrishnan, N. and Nagaraja, H.N. (2008). A First Course in Order Statistics, SIAM, Philadelphia.

Balakrishnan, N. and Li, T. (2006). Confidence Intervals for Quantiles and Tolerance Intervals Based on Ordered Ranked Set Samples, Annals of the Institute of Statistical Mathematics, 58, 757-777.

David, J.A. and Nagaraja, H.N. (2003). Order Statistics, Third edition, John Wiley, New York.

Emerson, J. (1982). Non-parametric Confidence Intervals for the Median in the Presence of Right Censoring, Biometrics, 38, 17-27.

Glick, N. (1978). Breaking Records and Breaking Boards, American Mathematical Monthly, $\mathbf{8 5}, 2-26$.

Gulati, S. and Padgett, W.J. (1994). Smooth Nonparametric Estimation of the Distribution and the Density Functions from Record Breaking Data, Communications in Statistics-Theory and Methods, 23, 1259-1274.

Gulati, S. and Padgett, W.J. (2003). Parametric and Nonparametric Inference from Record Breaking Data, Springer-Verlag, New York.

Guilbaud, O. (2001). Exact Non-parametric Confidence Intervals for Quantiles with Progressive Type-II Censoring, Scandinavian Journal of Statistics, 28, 699-713.

Krewski, D. (1976). Distribution-free Confidence Intervals for Quantile Intervals, Journal of the American Statistical Association, 71, 420-422.

Morabbi, H., Razmkhah, M. and Ahmadi, J. (2015). Point Estimation for Lower Quantiles Based on Two-sampling Scheme, Communication in Statistics-Theory and Methods, to appear. 
Nelson, W. and Doganaksoy, N., (1995). Statistical Analysis of Life or Strength Data from Specimens of Various Sizes Using the Power-(log) Normal Model. In Recent Advances in Life-Testing and Reliability, N. Balakrishnan, Ed, 377-408, CRC Press, Boca Raton.

Razmkhah, M., Ahmadi, J. and Khatib Astaneh, B. (2008), Nonparametric Confidence Intervals and Tolerance Limits Based on Minima and Maxima, Communications in StatisticsTheory and Methods, 37, 1525-1542.

Strawderman, R.L., Parzen, M.I. and Wells, M.T. (1997). Accurate Confidence Limits for Quantiles Under Random Censoring, Biometrics, 53, 1399-1415.

\section{H. Morabbi}

Department of Statistics, Ferdowsi University of Mashhad, Mashhad, Iran.

email:h.morabbi@gmail.com

\section{J. Ahmadi}

Department of Statistics, Ferdowsi University of Mashhad, Mashhad, Iran.

email:ahmadi-j@um.ac.ir

\section{Razmkhah}

Department of Statistics,

Ferdowsi University of Mashhad,

Mashhad, Iran.

email:razmkhah_m@um.ac.ir 